\title{
DESIGN OF A FLIGHT MANAGEMENT SYSTEM TO SUPPORT FOUR-DIMENSIONAL TRAJECTORIES
}

\author{
Manuel Angel AMARO CARMONA ${ }^{1}$, Darius RUDINSKAS ${ }^{2}$, Cristina BARRADO ${ }^{3}$ \\ ${ }^{1,3}$ Universitat Politècnica de Catalunya, Aerospace Science and Technology, Barcelona, Spain \\ ${ }^{2}$ Antanas Gustaitis Aviation Institue of Vilnius Gediminas Technical University, Rodunios kelias 30, \\ LT-02187 Vilnius, Lithuania \\ E-mails: ${ }^{1}$ manuel.angel.amaro@estudiant.upc.edu (corresponding author); ${ }^{2}$ darius.rudinskas@vgtu.lt; \\ ${ }^{3}$ cristina.barrado@upc.edu
}

Received 19 June 2014; accepted 20 January 2015

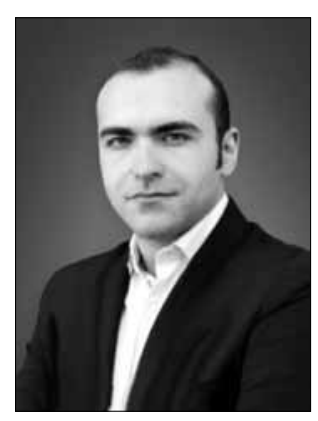

Manuel Angel AMARO CARMONA, MSc Eng.

Education: 2010 - engineer at Universidad de Los Andes, specialized in control and automation. 2014 - MSc at Aerospace Science and Technology of Universitat Politècnica de Catalunya.

Research interests: flight control and navigation, avionics systems and UAS.

Present position: master student at Cranfield University and Aerospace Research Engineer at CleanSky (SGO-GSAF).

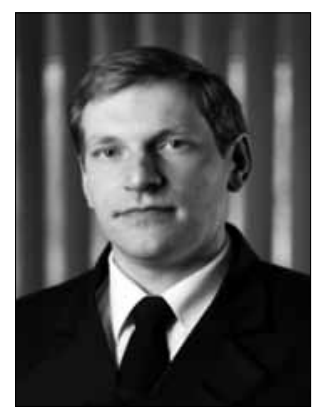

Darius RUDINSKAS, $D r$

Education: 2004 - electronic engineer at Vilnius Gediminas Technical University, Faculty of Electronics. 2006 - MSc electronic engineering, at Vilnius Gediminas Technical University, Faculty of Electronics. 2012 - PhD degree in measurement engineering, Antanas Gustaitis Aviation Institute of Vilnius Gediminas Technical University.

Research interests: wireless sensors, integrated health management systems, UAS, navigation and flight control systems.

Present position: PhD professor and deputy director at Antanas Gustaitis Aviation Institute.

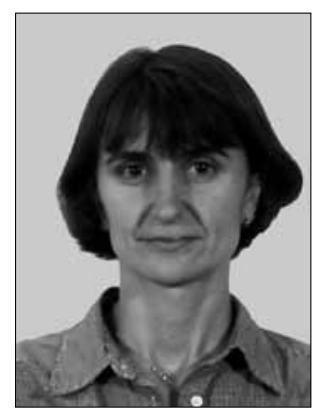

\section{Cristina BARRADO, $\mathrm{Dr}$}

Education: 1989 - computer science engineer, Faculty of Informatics, Universitat Politècnica de Catalunya. 1997 - PhD degree in computer sciences, Universitat Politècnica de Catalunya, Barcelona-Tech (Spain).

Research interests: UAS, navigation and flight control systems.

Present position: PhD professor and researcher in ICARUS Group.

Abstract. This paper presents the design and simulation of the functions of a flight Management System (FMS) in order to follow four-dimensional trajectories automatically. This is achieved by controlling the aircraft's airspeed, altitude, heading and vertical speed in order to arrive to the merging point in a specified time. The system receives data from the aircraft and computes new control parameters based on mathematical equations and algorithms of prediction trajectories. Additional features have been added to the FMS-4D, such as the capability of predicting the arrival time taking into account previous flight parameters and speed/altitude constrains. Finally, a testing phase was carried out using a flight simulator in order to obtain the performance and results of the designed system.

Keywords: FMS, 4D-trajectories, four dimensional navigation, control system. 


\section{Introduction}

Procedures and tasks related to air traffic management have been improved several years ago in order to increase the safety and reliability of air traffic. The concept of four dimension airspace management has become more prominent in the recent years due to work performed by international organizations with the objective of taking the airspace into a new dimension of performance and reliability.

According to Eurocontrol, the most-likely scenario C (Regulated Growth) has predicted up to 14.4 million flights in 2035 . This is equivalent to $50 \%$ more than in 2012 and has a growth average of $1.8 \%$ per year. This forecast alerts international organizations to the need to implement important improvements in airspace management. In view of this, a possible direction for improving air traffic transportation is the concept of $4 \mathrm{D}$ navigation (20-year... 2013).

$4 \mathrm{D}$ trajectory management is based on establishing in advance a sequence for all aircraft converging to a specific point in a congested area. This is achieved using trajectory predictions computed by air traffic management ground and airborne systems. The main idea is to provide each aircraft with a time constraint to get a specific merging point while allowing this aircraft to perform an autonomous flight in order to achieve this merging point in a given time (SESAR 2012).

If the merging point is not achieved in the specified time, the aircraft has to perform holding patterns in order to wait for a new time to achieve the merging point and to avoid disrupting the flight sequence predicted previously by the ground systems. Several problems are related to this fact. Firstly, an aircraft consumes more fuel which is converted to economic losses for the airline companies and even worse there are more $\mathrm{CO} 2$ emissions produced by this consumption Secondly, a new merging point sequence has to be computed by ground systems and this result in delays to the airlines as well as conflicts in airports.

Due to the issues discussed, it is important to provide aircraft with more accurate avionics systems capable to follow precise trajectories to achieve the specific merging point in the assigned time with a very low errors tolerance.

In this paper, the design of a Flight Management System (FMS) is described in order to support navigation procedures using four dimensional trajectories, as part of the improvements for future aircraft avionics and navigation systems necessary to the evolution of air traffic (Randy 2001).

\section{State of the art}

\subsection{Next generation flight management system (NG-FMS)}

The Next Generation Flight Management System or NGFMS is an avionic system developed and launched in
2010 by Honeywell $^{\odot}$ Inc. The system is based on performance that meets both the Single European Sky ATM Research (SESAR) and the NextGen Air Traffic Management (ATM) objectives (Sinclair 2010).

According to a study performed by R. Sabatini et al., the Next Generation Flight Management System (FMS) generates optimal trajectories (operationally and environmentally) due to mathematical models which were developed for this purpose (Sabatini et al. 2013). In the performance field, several cost functions have been considered in order to optimize fuel consumption, flight time and $\mathrm{CO}_{2}$ emissions. In addition, the models include aircraft dynamics, engine, atmosphere, noise and weather.

Other important features of the NG-FMS are the enabling of the Required Navigation Performance (RNP) 0.1, support of a Wide Area Augmentation System - Localizer Performance with Vertical guidance (WAAS-LPV), Future Air Navigation System 1 (FANS-1) and FANS-2 (Sinclair 2010).

This system is currently used by a few modern aircraft like the Boeing 747-8 as a stand-alone system and is integrated in other avionics systems of the Gulfstream G650.

\subsection{Flight management computer (FMC) and 10.7 GE aviation systems update}

Flight Management Systems used in the new generation of "Boeing B737-NG" (-600/700/800) are provided with a trajectory tracking function, which is a feature enabled by the 10.7 GE Aviation Systems Update (Klooster et al. 2009).

These FMS use ARINC 702A-1 Trajectory Bus which is a technology capable of sending information about the state of the aircraft every other minute or when the Flight Plan (FP) changes. The information includes latitude, longitude, altitude, turn radius, turn direction, fly-by waypoint type, and, of course, time. With this information, it is possible to obtain a full aircraft trajectory including vertical waypoints and turns.

The main idea of the use of this bus is to track this information and send it through an ACARS download data-link or output on a dedicated ARINC 429 in order to obtain a four-dimensional trajectory. The first trial flights utilizing this technology were performed in Sweden in a collaboration of SAS Airlines (Klooster et al. 2009).

\section{Theoretical model}

\subsection{Four dimensional navigation}

In order to perform navigation, it is necessary to know the initial waypoint and the final waypoint (and they are provided in the flight plan). From the mathematical point of view, a point in space is composed of three 
dimensional components ( $\mathrm{x}, \mathrm{y}$ and $\mathrm{z}$ ). However, when these points are used in order to represent a waypoint, its position with respect to the Earth is taken; this position is obtained according to its geographical coordinates.

Hence, a three dimension waypoint is composed of latitude, longitude and altitude.

Four dimensional navigation is based on reaching a three dimensional waypoint at a required time of arrival by changing the aircraft's flight profile. In this context several variables and parameters are involved in the concept of changing a flight profile. According to S. C. Mohleji, airspeed is considered as the most important control variable in order to achieve a waypoint at a given time of arrival (Mohleji 1989).

\subsection{Distance estimation}

Distance estimation is considered to be one of the most important parameters of the mathematical model for navigation and trajectory prediction. The Haversine equation for orthodromic distances provides simplicity for computational calculation and is used for navigation purposes.

The equation provides the orthodromic distance between two points in a sphere. As mentioned above, this equation is often used for navigation purposes due to its great precision for small distances (Sinnott 1984), because of this feature, the results can be used for the estimation of four-dimensional precision trajectories.

The Haversine equation can be divided into three main elements (Sinnott 1984).

The square of half the chord length between the two points denoted by:

$$
a=\sin ^{2}\left(\frac{\Delta \phi}{2}\right)+\cos \left(\phi_{1}\right) \cdot \cos \left(\phi_{2}\right) \cdot \sin ^{2}\left(\frac{\Delta \lambda}{2}\right) .
$$

The angular distance expressed (in radians) by:

$$
c=2 \cdot a \tan 2(\sqrt{a}, \sqrt{1-a}) .
$$

The orthodromic distance expressed (in kilometers) by:

$$
d=R \cdot c .
$$

\subsection{Bearing estimation}

A bearing refers to the direction that the aircraft have to move in order to reach a final point of a path. The bearing could change depending of the latitude of the initial and final point as well as the total distance of the path. Because of this, it is possible to compute an Initial Bearing and a Final Bearing.

However, when the distance between two points is really small, the Initial Bearing value is closer to the Final Bearing value (Williams 2014).
The equation of the initial bearing from a given initial point to a final point, in a straight line, along a greatcircle arc is denoted by the following equation:

$$
\begin{aligned}
& \theta=a \tan 2 \cdot\left(\sin (\Delta \lambda) \cdot \cos \left(\phi_{2}\right) \cdot \cos \left(\phi_{1}\right) \cdot \sin \left(\phi_{2}\right)-\right. \\
& \left.\sin \left(\phi_{1}\right) \cdot \cos \left(\phi_{2}\right) \cdot \cos (\Delta \lambda)\right) .
\end{aligned}
$$

\subsection{Time estimation}

The time that an aircraft spends moving from an initial point (lat, lon) to a final point (lat, lon) can be represented as follows:

$$
t=\frac{1}{\beta} \cdot \int_{x 1}^{x 2} \frac{\partial x}{V_{a}\left(z_{c}\right)} .
$$

where $V_{a}$ is the true airspeed of the aircraft at a given altitude and $\beta$ is a conversion factor from knots to feet/ seconds.

\subsection{Wind effect}

An important element that has to be taken into account for time computation is the along-track wind effect. Wind produces an important change of the airspeed of the aircraft and could affect the estimation of the time of arrival. The effect of the along-track wind over the aircraft's airspeed in cruise level is associated with two elements (Mohleji 1989):

The direction of the wind with respect to the aircraft. Depending on the relationship between the heading of the along-track wind and the aircraft, it is called either tailwind or headwind.

The magnitude of the along-track wind which represents the constant velocity of the wind at a given altitude (cruise altitude) (Table 1).

Table 1. Constants and variables of wind effect equations

\begin{tabular}{cl}
\hline Constant & \multicolumn{1}{c}{ Details } \\
\hline$V_{W}$ & Speed of the wind at a given altitude. \\
\hline$H_{W}$ & Heading of the wind at a given altitude. \\
\hline$\delta$ & $\begin{array}{l}\text { Factor to correct the magnetic north to true } \\
\text { north. }\end{array}$ \\
\hline$B$ & Bearing of the aircraft's track. \\
\hline
\end{tabular}

The along-track component of wind in the horizontal plane can be represented as follows (Mohleji 1989):

$$
w\left(z_{c}\right)=V_{w}\left(z_{c}\right) \cdot \cos \left(\left|B\left(z_{c}\right)-H_{w}\left(z_{c}\right) \pm \delta\right|\right) .
$$

By integrating the equation (5) into (6), the resultant equation used to compute the time of an aircraft's

\footnotetext{
1 The along-track component of the headwinds is negative and $s$ positive for tailwinds.
} 
flight between an initial point and a final point with constant velocity, is as follows:

$t=\frac{1}{\beta} \cdot \int_{x 1}^{x 2} \frac{\partial x}{V_{a}\left(z_{c}\right)+\left[V_{w}\left(z_{c}\right) \cdot \cos \left(\left|B\left(z_{c}\right)-H_{w}\left(z_{c}\right) \pm \delta\right|\right)\right]}$.

After integration of equation (7), the result is the time estimation equation with wind effect:

$t=\frac{1}{\beta} \cdot \frac{\left[x_{2}-x_{1}\right]}{V_{a}\left(z_{c}\right) \pm\left[V_{w}\left(z_{c}\right) \cdot \cos \left(\left|B\left(z_{c}\right)-H_{w}\left(z_{c}\right) \pm \delta\right|\right)\right]}$.

Note that this equation can be used in order to estimate the minimum time in which an aircraft can fly from the initial point to the final point at cruise airspeed. In addition, it is possible to isolate the airspeed in order to obtain the velocity that an aircraft should fly in order to arrive to the final point at a specific time, or it is even possible to isolate the factor $\left[x_{2}-x_{1}\right]$ to estimate the distance flown in a specific time at a given velocity.

This statement is important to understand the computation of flight control parameters explained in the next section.

\subsection{Vertical speed estimation}

Vertical speed is the rate of climb or descent of an aircraft. It can be estimated using the following equation:

$$
V S=\frac{\left|z_{1}-z_{2}\right|}{t} \cdot \frac{1}{\partial}
$$

a)

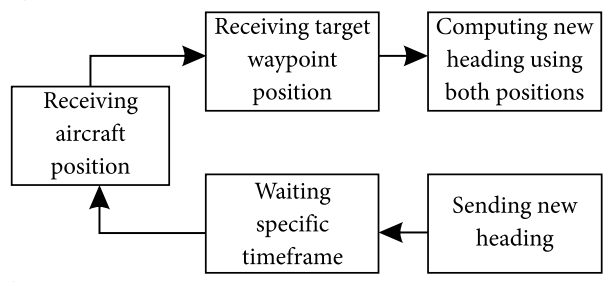

b)

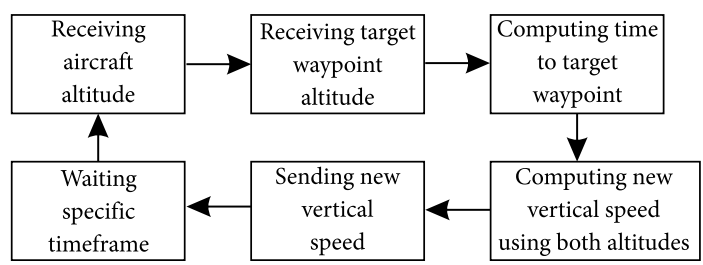

c)

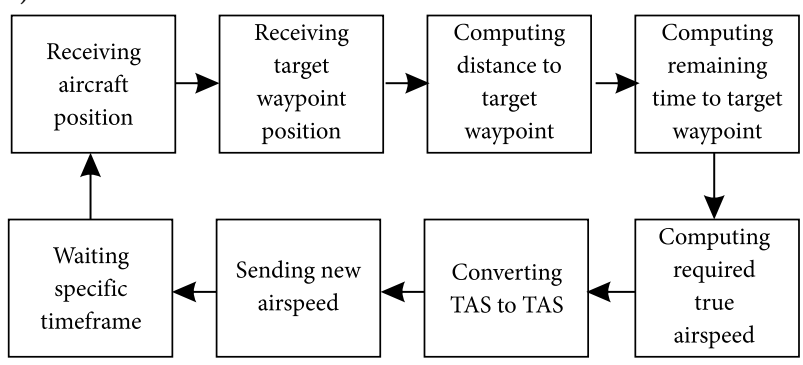

Fig. 1. a) - heading control; b) - altitude control; c) - airspeed control loops where $\partial$ is a conversion factor from seconds to minutes and $z_{1}-z_{2}$ is the difference of altitude between the initial point and the final point of the vertical trajectory.

The vertical speed is represented as a negative value when the aircraft is descending and a positive value is taken for the opposite case.

\section{Design}

\subsection{Heading control}

Heading control allows the aircraft to navigate correctly in the horizontal plane. The basic idea is to be able to compute the heading necessary to achieve a target waypoint (composed by latitude and longitude) from the current aircraft's position (composed by latitude and longitude as well).

To perform this task, the position of the aircraft is received and, by using equation (4) from the previous chapter, a new heading which is sent to the aircraft's autopilot panel is computed.

Figure 1 (a) shows the Heading Control performed by the FMS-4D for an aircraft.

\subsection{Vertical speed control}

In the same way that the aircraft uses the Heading Control in order to achieve a point in the horizontal plane, in the vertical plane, the situation is similar.

When the aircraft is flying in a constrained situation (e.g. below a constraint flight level), the vertical speed remains constant. This has been designed in order to obtain a correct prediction of the time when the aircraft crosses the constraint altitude.

On the other hand, when the aircraft is flying in a non-constrained situation (e.g. over a constraint flight level) the vertical speed is computed using equation (9) and vertical speed control is performed, as illustrated by the following diagram.

\subsection{Airspeed control}

Airspeed control is considered to be the core of flight control regarding four-dimensional navigation. The airspeed is the most important parameter to validate that the aircraft has arrived at a target waypoint at an expected arrival time.

The airspeed of an aircraft is sensitive to different factors. When an aircraft is ascending, the airspeed tends to decrease and increases, when it is descending. In addition, an aircraft's airspeed is modified by the flaps position, airbrakes and landing gear. However, most of these changes can be controlled with a change in the aircraft thrust, which is transferred directly as a change of the aircraft's airspeed.

Equation (8) expresses the time that passes when an aircraft is flying a known distance at a given airspeed. The main idea of airspeed control is based on the fact 
that time and distance are known parameters ${ }^{2}$; therefore, it is possible to obtain the required true airspeed of the aircraft needed to arrive at the target waypoint at a defined arrival time.

The airspeed obtained by equation (8) refers to true airspeed (TAS). However, the airspeed sent to the aircraft is the indicated airspeed (IAS). Therefore, true airspeed (TAS) is converted to the indicated airspeed (IAS), before sending it to the aircraft, using the density information provided by the variables of the simulator (X-Plane... 2013).

In addition to the routines shown in (c), a proportional controller feature has been added. The main idea is to improve airspeed computation with respect to the delays made by the aircraft (airspeed changes slower than other parameters, such as vertical speed and heading).

\subsection{Speed and altitude constraints}

The main idea behind speed constraints is that the FMS-4D performs a check before computing a required airspeed. If the flight level is below the constraint flight level, then the airspeed is not computed using the airspeed control loop but remains constant at a constraint airspeed (except when the airspeed control loop determines that the airspeed has to be lower than the constraint airspeed).

The Figure 2 shows the typical flow performed during airspeed control in order to follow the airspeed constraint.

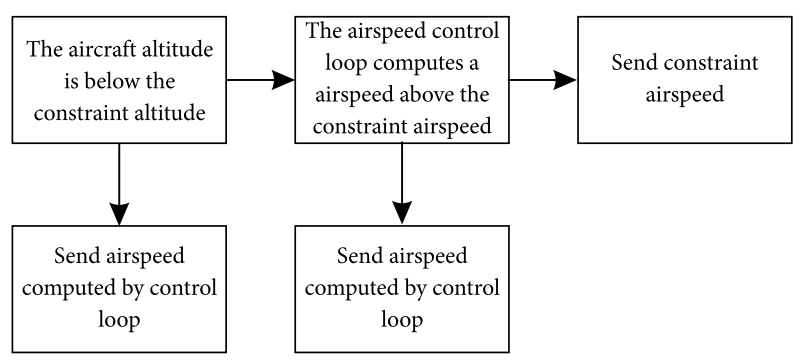

Fig. 2. Speed/altitude check

\subsection{Minimum arrival time prediction}

What would happen if the ATM Ground System assigned an arrival time that cannot be achieved by the aircraft due to technical limitations or due to speed/altitude constraints?

This section discusses the issue presented above, by explaining one of the key features of the FMS-4D: the way to compute a Minimum Predicted Arrival Time (PAT).

The Predicted Arrival Time (PAT) is a parameter designed for this FMS-4D which gives the pilots an idea of the best performance of an aircraft. This parameter

\footnotetext{
2 The time is computed continuously and the distance is computed using the Haversine equation (3).
}

informs the crew about the minimum time that is required for an aircraft to achieve a target waypoint. By using this information, the crew could discuss with the ATC if it is possible to be assigned a specific arrival time or if this time has to be delayed instead.

When a new waypoint is added to the FMS-4D, a new PAT for that waypoint is computed taking into account the following parameters and/or situations:

- maximum aircraft cruise speed,

- the existence of speed/altitude constraints.

If there are no speed/altitude constraints in the trajectory, the PAT is computed using equation (8). For this case, the distance is computed using equation (3) and the speed is a constant parameter equivalent to the maximum aircraft cruise speed ${ }^{3}$.

If there is a speed altitude constraint for a trajectory, it is necessary to compute the PAT by using the following equation $^{4}$ :

$$
P A T=P A T_{C S}+P A T_{N C S}
$$

Where $P A T_{C S}$ is the predicted arrival time from the initial position to the point where the constraint altitude $(C A P)$ is achieved, and $P A T_{N C S}$ is the predicted arrival time from the point where the constraint altitude $(C A P)$ is achieved to the target waypoint. Hence, the trajectory of the aircraft is divided into two sections as shown in Figure 3.

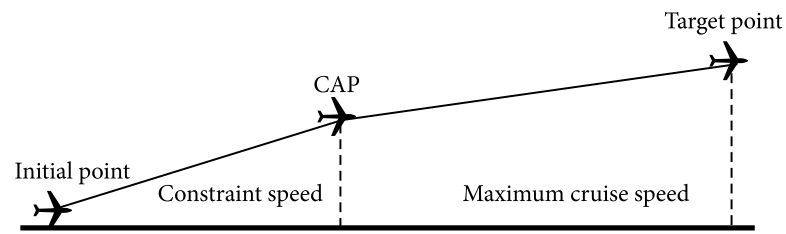

Fig. 3. Trajectory of the aircraft used to compute PAT

Initially, with the conditions of a normal flight controlled by the FMS-4D and equations explained in the previous sections, it is not possible to know the exact components of the CAP point (latitude and longitude) or the distance from the CAP point and the Target Point.

In order to solve this problem, it has been agreed that the vertical speed could be defined as a constant parameter according to aircraft specifications, since aircraft airspeed is constant in most of the cases while flying before the CAP point.

Hence, by knowing the vertical speed and the airspeed at which the aircraft would be flying from Initial

3 The maximum cruise speed is a parameter defined in the FMS$4 \mathrm{D}$ and depends on the aircraft model, and other technical specifications.

4 This equation is used for any situation where the aircraft trajectory involves speed/altitude constraints. Despite of the fact that Figure 4 shows an ascending situation, this procedure is applied to descending situations as well. 
Point to CAP, it is possible to predict the time when the aircraft crosses the constraint flight level and distance at that point (Fig. 3).

This means that the $P A T_{C S}$ is calculated using the constant vertical speed as follows:

$$
P A T_{C S}=\frac{\left|Z_{I P}-Z_{C A P}\right|}{V S_{C S}} \cdot \frac{1}{\partial},
$$

where $Z_{I P}$ is the altitude at the initial point, $Z_{C A P}$ is the constraint altitude and $V S_{C S}$ is the constant vertical speed defined by aircraft specifications. Also $\partial$ is the conversion factor from minutes to seconds for the vertical speed.

Once the $P A T_{C S}$ is computed, it is necessary to compute the other component of equation (10).

Then, in order to calculate the value of $P A T_{N C S}$, it is necessary to compute the distance from CAP to the Target Point as follows:

$$
D_{\text {CAPtoTP }}=D_{\text {total }}-D_{\text {IPtoCAP }}
$$

where $D_{\text {total }}$ is a well-known parameter which is calculated using the Haversine equation (3) and $D_{\text {IPtoCAP }}$ is calculated using equation (8), isolating the distance $\left[x_{2}-x_{1}\right]$ and replacing the time with the $P A T_{C S}$.

Finally, the value of $P A T_{C S}$ is calculated using again equation 8 while using the Maximum Cruise Speed of the aircraft.

The control functions use the information provided by the input parameters in order to produce new output

\begin{tabular}{|c|c|c|}
\hline $\begin{array}{c}\text { Input } \\
\text { parameters } \\
\text { (from aircraft } \\
\text { sensors) }\end{array}$ & $\begin{array}{c}\text { Control } \\
\text { functions } \\
\text { (computes new } \\
\text { parameters) }\end{array}$ & $\begin{array}{c}\text { Output } \\
\text { parameters } \\
\text { (to autopilot } \\
\text { functions) }\end{array}$ \\
\hline $\begin{array}{l}\text { - Speed } \\
\text { - Altitude } \\
\text { - Latitude } \\
\text { - Longitud }\end{array}$ & $\begin{array}{l}\text { - New HDG } \\
\text { - New SPD } \\
\text { - New VS } \\
\text { - New Alt } \\
\text { - New CTA }\end{array}$ & $\begin{array}{l}\text { - HDG } \\
\text { - SPD } \\
\text { - VS } \\
\text { - ALT }\end{array}$ \\
\hline
\end{tabular}
parameters, as shown in the following Figure 4.

Fig. 4. System scheme

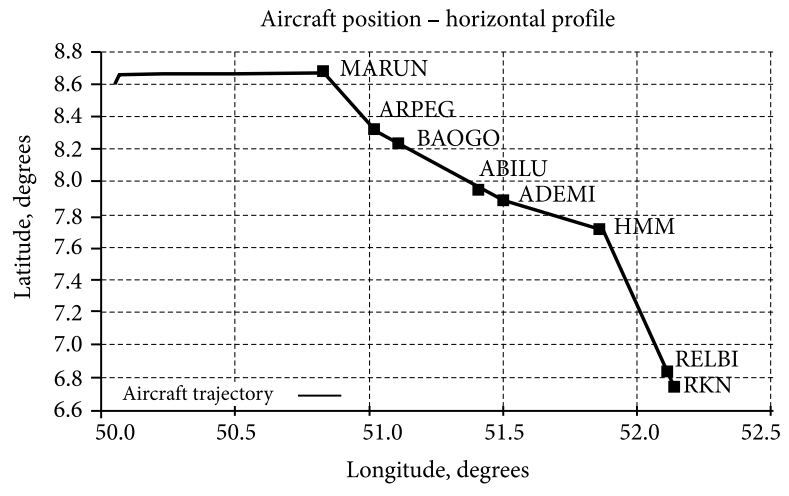

Fig. 5. Horizontal plane navigation (Heading control)

\section{Test and results}

Specific tests in order to know the general behavior of the FMS-4D algorithms have been designed and performed. Taking into account the requirements for the test, the route from Frankfurt Main Airport (EDDF) to Amsterdam Schiphol Airport (EHAM) was chosen.

The automatic flight performed by the FMS-4D has been analysed between the waypoints MARUN (beginning of ascending phase) to seconds before REKKEN (beginning of approach phase).

\subsection{Horizontal plane navigation (heading control)}

The Latitude [deg] vs. Longitude [deg] plot represents the horizontal navigation behavior of the aircraft. Constant changes of the aircraft's heading used to achieve the flight plan waypoints in an efficient way are evident.

In the test simulation, the aircraft maintained a heading with very accurately. When a new target waypoint is followed, the heading of the aircraft changes ${ }^{5}$ instantly to the new target. Slow changes in the output of the heading control system have been detected a few seconds after turning and intercepting the predicted course.

The following Figure 5 shows the changes of the heading performed by the aircraft during the whole simulation period, as well as a brief overview of the path followed during the simulation.

Results of the Minimum Distance to the Target Waypoint are shown in Table 2. According to this information, the performance of the FMS in the horizontal plane has been achieved with a very low error in the route path. The maximum error is below 0.5 nautical miles and the minimum is around 0.3 nautical miles.

The heading control of the FMS-4D allows the aircraft to pass through all the Flight Plan waypoints with an acceptable low error.

Table 2. Results of minimum distance to the target waypoint

\begin{tabular}{cc}
\hline Target waypoint & Minimum distance $(\mathrm{nm})$ \\
\hline MARUN & 0.36793 \\
\hline ARPEG & 0.32781 \\
\hline BADGO & 0.34483 \\
\hline ABILU & 0.49713 \\
\hline ADEMI & 0.49901 \\
\hline HMM (HAMM) & 0.38090 \\
\hline REGBU & 0.39680 \\
\hline RELBI & 0.41239 \\
\hline REKKEN $($ RKN) & - \\
\hline
\end{tabular}

$\overline{5}$ The bank angle of the aircraft has been set to 30 degrees showing a normal performance during the whole simulation. 


\subsection{Vertical plane navigation (vertical speed control)}

The Altitude [ft] vs. Absolute Time [sec] plot represents the aircraft's behavior during vertical navigation (Fig. 6). The FMS-4D controlled the aircraft so that it would achieve the requested altitude for all the waypoints in a smooth and regular way in the time required.

In Figure 6 between 0 and MARUN a two-step ascending trajectory can be clearly identified as studied in section 4.5. Minimum arrival time prediction and as illustrated in Figure 3 of the same section. The point at 11.000 feet represents the CAP point. In the segment before this point, the vertical speed and the speed remained constant at 3000 feet per min and, in the segment after the CAP point till MARUN, the vertical speed changed slightly according to the computation performed by the vertical speed control.

In addition, in the ascending phase, three phases with different climb rates can be clearly defined: from the start of the ascending point to MARUN (FL200), from MARUN to BADGO (FL30000) and from BADGO to ABILU/Cruise Level (FL320). The change of vertical speed to reach the desired altitude in the exact time required is evident from the results.

In the cruise phase, the aircraft maintained FL320 with small changes of a few feet due to the normal oscillation produced by the non-uniform air density or wind effects simulated by X-Plane and the altitude control adjustment was performed automatically by the autopilot.

\subsection{Precision time/distance (airspeed control)}

As discussed in previous sections, if the waypoint is reached too early or too late (according to the required time), this could result in the necessity of performing holding patterns in order to wait for another available time to arrive at the target waypoint.

The relative distance decreases, and, accordingly, the simulation time gets closer to the required time of arrival.

When the simulation point equals the required time, the distance to the target waypoint is almost zero. Table 3 shows how the arrival time matches accurately with the predicted arrival time (PAT) ${ }^{6}$.

As shown in the previous table, the maximum error obtained was 3 seconds and the minimum was 2 seconds. In all the waypoints, the aircraft arrived before the required arrival time.

As a result, true airspeed varies in a range which does not exceed \pm 15 knots of the defined cruise airspeed (350 knots). The continuous change of airspeed is the main factor that allows the aircraft to meet the Prediction Arrival Time (PAT) with very low errors.

\footnotetext{
6 The Predicted arrival time and arrival time shown in the Table 3 are measured with respect to the simulation starting point (simulation time $=0$ ).
}

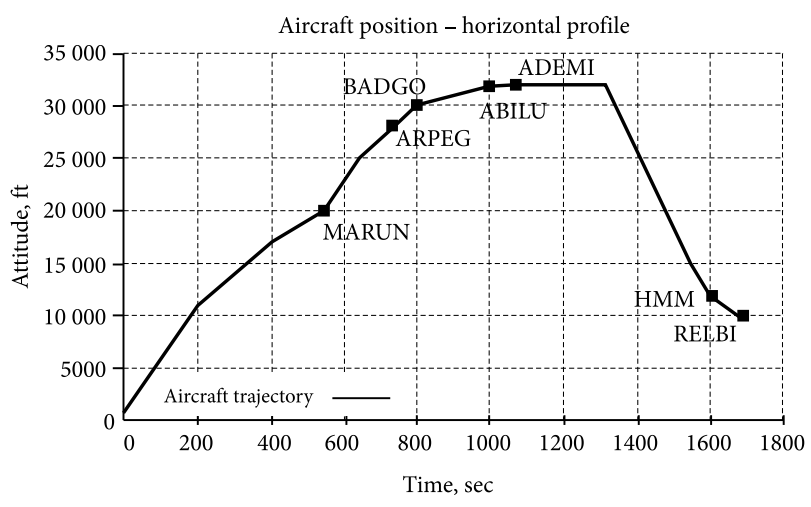

Fig. 6. Vertical plane navigation (Vertical speed control)

Table 3. Comparison of arrival time with respect to the predicted arrival time, relative to the previous target waypoint

\begin{tabular}{cccc}
\hline Waypoint & $\begin{array}{c}\text { Predicted } \\
\text { arrival time }\end{array}$ & Arrival time & Error \\
\hline MARUN & $550(9: 13)$ & 548 & $2(-)$ \\
\hline ARPEG & $737(12: 23)$ & 735 & $2(-)$ \\
\hline BADGO & $793(13: 16)$ & 791 & $2(-)$ \\
\hline ABILU & $1015(17: 14)$ & 1012 & $3(-)$ \\
\hline ADEMI & $1067(18: 11)$ & 1064 & $3(-)$ \\
\hline HMM & $1308(21: 11)$ & 1306 & $2(-)$ \\
\hline REBGU & $1612(26: 16)$ & 1610 & $2(-)$ \\
\hline RELBI & $1688(28: 02)$ & 1685 & $3(-)$ \\
\hline RKN & $1713(29: 14)$ & - & - \\
\hline
\end{tabular}

\section{Conclusions}

In order to provide a possible solution to the problems faced by aircraft when following a four-dimensional trajectory, a software-based Flight Managed System (FMS) that performs the control of the flight in order to achieve three dimensional waypoints in the required arrival time estimated has been designed.

The designed system is composed of control functions that take parameters defining the flight status as input and uses mathematical equations and estimation algorithms in order to calculate new values for heading, vertical speed and airspeed. This way the aircraft is able to accurately follow a four dimensional trajectory defined by the Flight Plan (FP).

Also, the FMS-4D calculates another parameter defined as the Prediction Arrival Time (PAT), which is an estimation of the time of arrival that takes the aircraft to fly a specific path. The PAT is used to inform the crew about the estimated performance of the aircraft, so that this information could be used to discuss the possibility of time constraints with air traffic management services. 


\section{References}

20-year Forecast of Annual Number of IFR Flights (2012-2035) 2013. EUROCONTROL.

Klooster, J. K.; Del Amo, A.; Manzi, P. 2009. Controlled timeof-arrival flight trials, in Eighth USA/Europe Air Traffic Management Research and Development Seminar, 29 June02 July 2009, Napa, California, USA.

Mohleji, S. C. 1989. Optimal flight management system utilization with ATC automation, IEEE Aeropostale and Electronic Systems Magazine 4(2): 26-32. http://dx.doi.org/10.1109/62.16993

Randy, W. 2001.The Avionics Handbook: Flight Management System. Smith Industries by CRC Press LLC.

Sabatini, R.; Ramasamy, S.; Gardi, A., et al. 2013. Novel Four Dimensional Flight Management System With Enhanced Navigation Integrity Monitoring. Institute of Navigation GNSS.

SESAR 2012. SESAR Factsheet: I-4D - Flying a New Dimension. Single European Sky Research Team (SESAR). (01).

Sinclair, C. 2010. Honeywell Next Generation Flight Management System Meets New Air Traffic Requirements [online]. Honeywell Aerospace Media Center [cited 26 January 2014]. Available from Internet: http://www51. honeywell.com/honeywell/news-events/press-releases-details/03.04.10HONNextGenNewAirTrafficRequirements. html

Sinnott, R. W. 1984. Virtues of the haversine, Sky and Telescope 68(2): 158.

Williams, E. 2014. Aviation Formulary V1.33 [online], [cited 26 January 2014]. Available from Internet: http://williams. best.vwh.net/avform.htm

X-Plane Documentation 2013. Data Input \& Output. Laminar Research [online]. Chapter 6, section 6.1 [cited 26 January 2014]. Available from Internet: http://wiki.x-plane.com/ Chapter_5:_X-Plane_Menus\#Data_Input_.26_Output 